\title{
PoliFi: Airtime Policy Enforcement for WiFi
}

\author{
Toke Høiland-Jørgensen \\ Dept. of Computer Science \\ Karlstad University, Sweden \\ toke.hoiland-jorgensen@kau.se
}

\author{
Per Hurtig \\ Dept. of Computer Science \\ Karlstad University, Sweden \\ per.hurtig@kau.se
}

\author{
Anna Brunstrom \\ Dept. of Computer Science \\ Karlstad University, Sweden \\ anna.brunstrom@kau.se
}

\begin{abstract}
As WiFi grows ever more popular, airtime contention becomes an increasing problem. One way to alleviate this is through network policy enforcement. Unfortunately, WiFi lacks protocol support for configuring policies for its usage, and since network-wide coordination cannot generally be ensured, enforcing policy is challenging.

However, as we have shown in previous work, an access point can influence the behaviour of connected devices by changing its scheduling of transmission opportunities, which can be used to achieve airtime fairness. In this work, we show that this mechanism can be extended to successfully enforce airtime usage policies in WiFi networks. We implement this as an extension our previous airtime fairness work, and present PoliFi, the resulting policy enforcement system.

Our evaluation shows that PoliFi makes it possible to express a range of useful policies. These include prioritisation of specific devices; balancing groups of devices for sharing between different logical networks or network slices; and limiting groups of devices to implement guest networks or other low-priority services. We also show how these can be used to improve the performance of a real-world DASH video streaming application.
\end{abstract}

\section{INTRODUCTION}

WiFi is increasingly becoming the ubiquitous connectivity technology in homes as well as in enterprises. The ability for anyone to set up an access point and connect any device to it is one of the driving factors behind this increase of popularity. However, increased popularity also means increased contention for resources as more devices are deployed.

Since no two devices can transmit at the same time on a given frequency, the sparse resource that determines performance in WiFi networks is the time spent transmitting, also known as airtime usage. The 802.11 Media Access Control (MAC) protocol used in WiFi networks does not, in itself, guarantee a fair usage of this sparse resource. In fact it is well known that devices transmitting at lower rates can use more than their fair share of the airtime [1].

One way to improve performance of a network under contention is to apply different policies to different devices on the network, which works best if applied directly to the sparse resource instead of a proxy such as byte-level throughput. However, WiFi is decentralised at the protocol level, and thus lacks protocol support for enforcing policies on airtime usage. Fortunately, it turns out that in the common infrastructure deployment scenario, the access point can exert quite a bit of influence on the transmission behaviour of clients, or stations, as they are commonly called. In previous work, we have shown that this makes it possible to achieve airtime fairness between stations in a WiFi network by making appropriate scheduling decisions at the AP [2]. Given such a mechanism to enforce fairness, a natural question is whether it can be extended to express different capacity sharing policies. In this work we answer this question in the affirmative, in the form of a workable solution to airtime policy enforcement in $\mathrm{WiFi}$, which we have named PoliFi.

The number of possible policies one might want to express is all but infinite. Therefore, to focus our discussion, we define the following three representative policy use cases:

1) Prioritising devices. It should be possible to configure one or more devices to receive a higher share of network resources than other devices on the network.

2) Balancing device groups. In this use case, the network should be configured to share the available resources between groups of devices in a given way. For instance, this could be used to implement the "network slicing" concept often seen in 5G architectures [3].

3) Limiting groups of devices to a maximum capacity share, in order to implement a lower-priority service, such as a guest network.

PoliFi makes it possible for the user to express all of these policies. Our design builds on our previous airtime scheduler for the Linux kernel, but extends it by (a) generalising the implementation from a specific driver to the common kernel WiFi stack, (b) extending the kernel scheduler to support weighted scheduling of stations, and (c) adding a userspace policy daemon that transforms the higher-level policy decisions into configuration of the kernel scheduling mechanism.

The rest of this paper presents PoliFi in detail, and is structured as follows: Section II summarises related work. Section III describes our design, with a performance analysis presented in Section IV. Finally, Section V concludes.

\section{RELATED WORK}

Network policies are, in general, nothing new. For instance, standardisation of different traffic classes has occurred in the form of the DiffServ framework [4]. In the WiFi world, the 802.11e standard defines different priority levels, which can be mapped to DiffServ code points [5]. However, this is all related to applying policies to different types of traffic, whereas PoliFi deals with realising different capacity sharing policies between devices on the same network at the airtime usage level. As such, PoliFi is orthogonal so DiffServ, 802.11e and other traffic class policy mechanisms. 
As mentioned above, PoliFi is an extension of our previous work implementing an airtime fairness enforcement mechanism in Linux [2]. Compared to this previous work, PoliFi adds the policy enforcement component, and also generalises the mechanism by moving it out of the device drivers and into the common WiFi subsystem in Linux, thus making it applicable to more device drivers.

The concept of airtime policy enforcement appears in the concept of network slicing, which is an important part of the upcoming 5G mobile network architecture [3]. Network slicing involves splitting up a network into several virtual parts that are conceptually isolated from one another, which is a form of policy enforcement. A description of how to achieve network slicing in WiFi networks is given in [6], which corresponds roughly to our second use-case. The authors implement a prototype in simulation. Our mechanism builds on the same basic concept of computing per-device weights from group weights, but we solve a number of issues that prevent it from being implemented on real hardware. In addition, [6] only covers the second of our three policy use-cases.

Another approach to splitting a wireless network into multiple parts is presented in [7], which describes a scheme where a separate software router is installed in the access point. This software router queues packets and enforces capacity sharing. However, the capacity sharing is implemented at the bandwidth level which, as mentioned above, is not the sparse resource in a WiFi network.

A description of a scheme for network slicing in a home network is described in [8]. The authors describe a design that uses Software Defined Networking (SDN) to split a home network into different parts, but do not discuss any mechanism for how the sharing is achieved.

Finally, some enterprise APs offer features related to airtime fairness and policy configuration, e.g., [9]. Unfortunately, no technical description of how these policies are enforced is generally available, which prevents us from comparing them to our solution.

\section{The Polifi Design}

We have designed PoliFi as a two-part solution, where a user-space daemon is configured by the user, and in turn configures a scheduling mechanism in the kernel. In this section, we describe our design in detail. A diagram of the design is shown in Figure 1. We begin by describing the user space daemon that configures the policies. Following this, we describe how the weighted Deficit Round-Robin (DRR) scheduling mechanism is used to achieve the desired policies, and finally we describe how the mechanism is integrated into the Linux kernel WiFi stack.

\section{A. Userspace Policy Daemon}

We implement the userspace policy daemon as part of the hostapd access point management daemon. This is the daemon responsible for configuring wireless devices in access point mode in Linux. This means it already implements policies for other aspects of client behaviour (such as authentication),

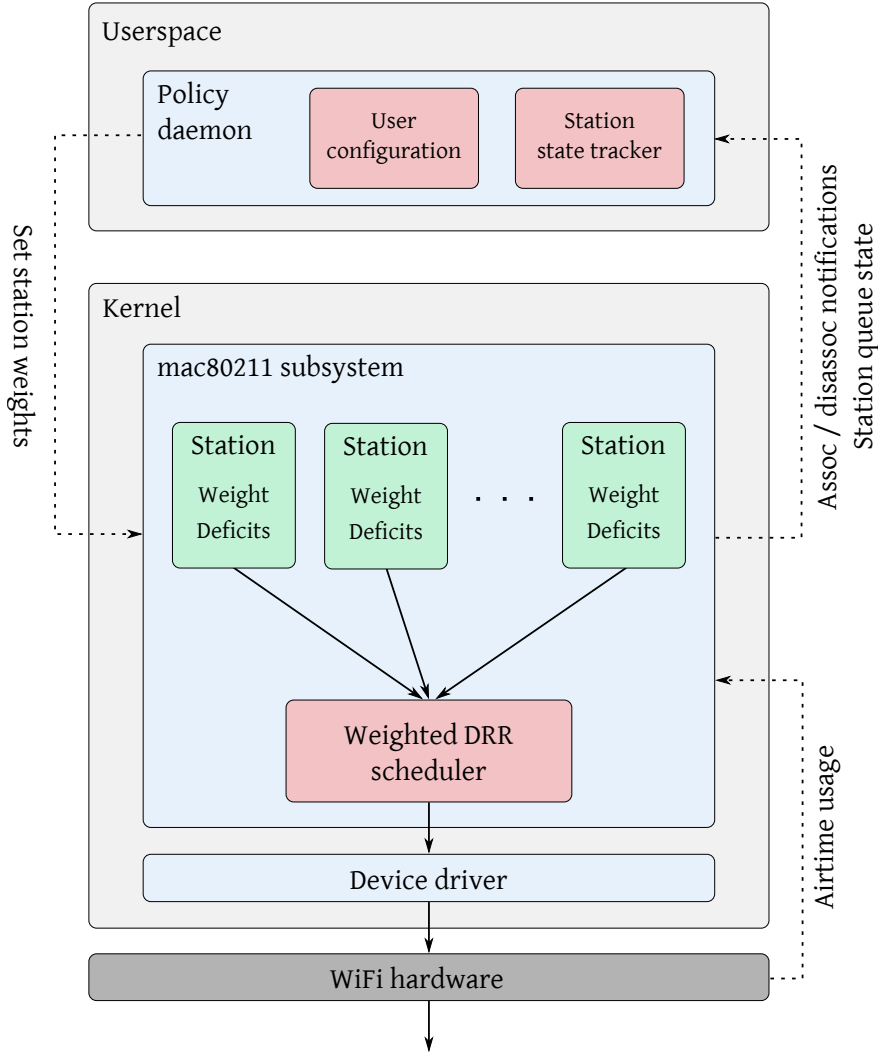

Fig. 1: The high-level design of PoliFi. The kernel maintains data structures for every station, containing its current airtime deficits and configured weight. The scheduler uses this to decide which station to transmit to next. The hardware reports airtime usage on TX completion. The userspace daemon tracks the associated stations and their queue state, and updates the weights in the kernel based on user policy preferences.

which makes it a natural place to implement airtime policy as well.

The module we have added to hostapd can be configured in three modes, corresponding to the three use cases described in the introduction: static mode, dynamic mode and limit mode. The user can configure each of these modes per physical WiFi domain, and assign parameters for individual stations (based on their MAC addresses), or for entire Basic Service Sets (BSSes). The latter is a natural grouping mechanism, since this corresponds to logical networks configured on the same device (e.g., a primary and a guest network). However, extending the design to any other logical grouping mechanism is straight forward.

In static mode, the daemon will simply assign static weights to stations when they associate to the access point. Weights can be configured for individual stations, while a default weight can be set for each BSS, which will be applied to all stations that do not have an explicit value configured. This implements the basic use case of assigning higher priorities to specific devices, but does not guarantee any specific total share.

The dynamic and limit modes work by assigning weights 
to each BSS, which are interpreted as their relative shares of the total airtime, regardless of how many stations they each have associated. Additionally, in limit mode, one or more BSSes can be marked as limited. BBSes that are marked as limited are not allowed to exceed their configured share, whereas no limitations are imposed on unmarked BSSes. Thus, dynamic mode implements the second use case, while limit mode implements the third.

For both dynamic mode and limit mode, the daemon periodically polls the kernel to discover which stations are currently active, using the queue backlog as a measure of activity, as discussed below. After each polling event, per-station weights are computed based on the number of active stations in each BSS, and these weights are configured in the kernel. The details of the weight computation, and how this is used to achieve the desired policy, is discussed in the next section. Selecting the polling frequency is a tradeoff between reaction time and system load overhead. The polling interval defaults to $100 \mathrm{~ms}$, which we have found to offer good reaction times (see Section IV-B), while having a negligible overhead on our test system.

While our implementation is focused on the single access point case, where the access point enforces a single configured policy, the userspace daemon could just as well pull its policy configuration from a central cloud-based management service, while retaining the same policy enforcement mechanism.

\section{B. Weighted Airtime DRR}

The fairness mechanism that we are starting from (described in detail in [2]) is a Deficit Round-Robin scheduler, which operates by accounting airtime usage as reported by the WiFi hardware after a transmission has completed, and scheduling transmissions to ensure that the aggregate usage over time is the same for all active stations. Using the airtime information provided after transmission completes means that retransmissions can be accounted for, which improves accuracy especially for stations with low signal quality. Furthermore, as we have shown in our previous work, for TCP traffic we can provide fairness even for transmissions transfers coming from each station. This is achieved by accounting the airtime of received packets, which causes the scheduler to throttle the rate of TCP ACKs going back to the station.

1) Adding Weighted Scheduling: Given this effective airtime fairness scheduler, we can realise arbitrary division of the available capacity between different stations, by simply assigning them different scheduling weights. For the DRR scheduling algorithm employed by our scheduler, this is achieved by using different quantums per station. Thus, to apply this to airtime policy enforcement, we need to express the desired policy as a number of different service weights for each of the active devices.

The first use case is trivially expressed in terms of weights: simply assign the prioritised device a higher weight; for instance, to double its priority, assign it a weight of 2 . The second use case, where capacity should be split between groups of devices has been covered in the network slicing use case described in [6]: each group is assigned a weight signifying its share relative to the other groups; from these group weights, each device in that group is assigned a weight computed by dividing the group weight with the number of active devices in that group.

The final use case requires limiting one or more groups of stations to a fixed share of the available capacity. This can be illustrated with the guest network use case, where an example policy could be that a guest network is not allowed to exceed $50 \%$ of the available capacity. If this policy is implemented as a fixed share between groups, however, a single station on the guest network would be able to get the same capacity as, say, five users of the regular network, which is not what we want. Thus, a different policy is needed: a group can be limited, and should have its weight adjusted only if it would get more than the configured share, not if it gets less. Thus, this becomes a two-step procedure that first assigns unit weights to all devices (which is the default when no policy is applied), and calculates whether or not this results in the limited group using more than its configured share of the airtime. If it does, a policy is computed in the same way as for the dynamic use case, which results in the limited group being assigned exactly its configured airtime share.

2) Computing the Weights: Having established that our desired policies can be expressed in terms of weights, we turn to the practical difficulties of applying this to a real $\mathrm{WiFi}$ system.

First, the approach outlined above assumes that we have knowledge of which stations are active at any given time. This might look trivial at first glance, since an access point needs to maintain some amount of state for all currently associated clients in any case. However, clients can be associated to an access point without sending or receiving any data, and thus without consuming any airtime. This means that association state in itself is not sufficient to ascertain the set of currently active clients. Fortunately, we have another piece of data: The queue backlog for each device. Monitoring the backlog gives us a straight-forward indicator for activity without having to monitor actual packet flows; we can simply consider any device that has had a non-zero queue backlog within a suitably short time span as active, and use that number in our calculations.

The second difficulty lies in the fact that we need to transform the total weights between groups of stations into weights for each individual station. As shown in [6], this is conceptually just a simple division. However, when implementing this in an operating system kernel, we are limited to integer arithmetic, which means that to get accurate weights, we need to ensure that the division works when confined to the integers. To achieve this, we first limit our configuration language to be expressed as integer weights between groups. Then, to ensure that we can divide these weights with the number of active stations, we multiply them by a suitable constant, chosen as follows:

We are given the set of groups $I$, where each group $i$ has a configured group weight $W_{i}$ and $N_{i}$ active stations. We then 
define the multiplication constant $C=\prod_{i \in I} N_{i}$. Multiplying all group weights by this same constant maintains their relative ratio, and the choice of constant ensures that each group's weight can be divided by the number of active stations in that group. This gives us the following expression for the perstation weight for group $i$ :

$$
W_{i}^{s}=\frac{W_{i} C}{N_{i}}
$$

The third issue we need to deal with is converting the weights to the per-station time quantums that are used in the scheduler, and which are expressed in microseconds. These should be kept at a reasonable absolute size, because larger weights result in coarser time granularity of the scheduler, making each scheduler round take longer and impacting latency of all devices in the network. We convert the calculated weights into final quantums by normalising them so they fall within a range of $100-1000 \mu s$, but preferring smaller values if the ratio between the smallest and largest weight is more than $10 \times$.

\section{Kernel Airtime Scheduler}

We implement the kernel part of PoliFi in the WiFi protocol subsystem of the Linux kernel (called mac80211). Our implementation builds on our previous airtime fairness scheduler, described in [2], which implemented a queueing system in this layer. In this queueing system, packets are assigned a Traffic ID (TID) before enqueue, and a separate queueing structure is created for each TID, of which there are 16 per station. These per-TID queues then form the basis of the scheduling of different stations. The queueing structure itself is based on the FQ-CoDel queue management scheme [10] and ensures flow isolation and low queueing latency.

While our previous implementation implemented queueing in the general WiFi layer, scheduling and tracking of each active station's airtime usage was still the responsibility of the driver. In PoliFi, we move the scheduling decisions into mac80211, where it can be leveraged by all device drivers. In addition, we modify the scheduler to support the weightbased policy enforcement capability described above. The weights can be set by userspace through the standard $n l 80211$ configuration API.

In order to move the scheduling decision out of the drivers, we define a new driver API, shown in Algorithm 1. The device driver runs the schedule() function, and asks mac80211 for the next TID queue to schedule, using the get_next_tid() API function. The driver then services this queue until no more packets can be scheduled (typically because the hardware queue is full, or the TID queue runs empty). After this, the driver uses the return_tid() API function to return the TID queue to the scheduler. A third API function, account_airtime (), allows the driver to register airtime usage for each station, which is typically done asynchronously as packets are completed or received.

Using this API, mac80211 has enough information to implement airtime policy enforcement using the weighted

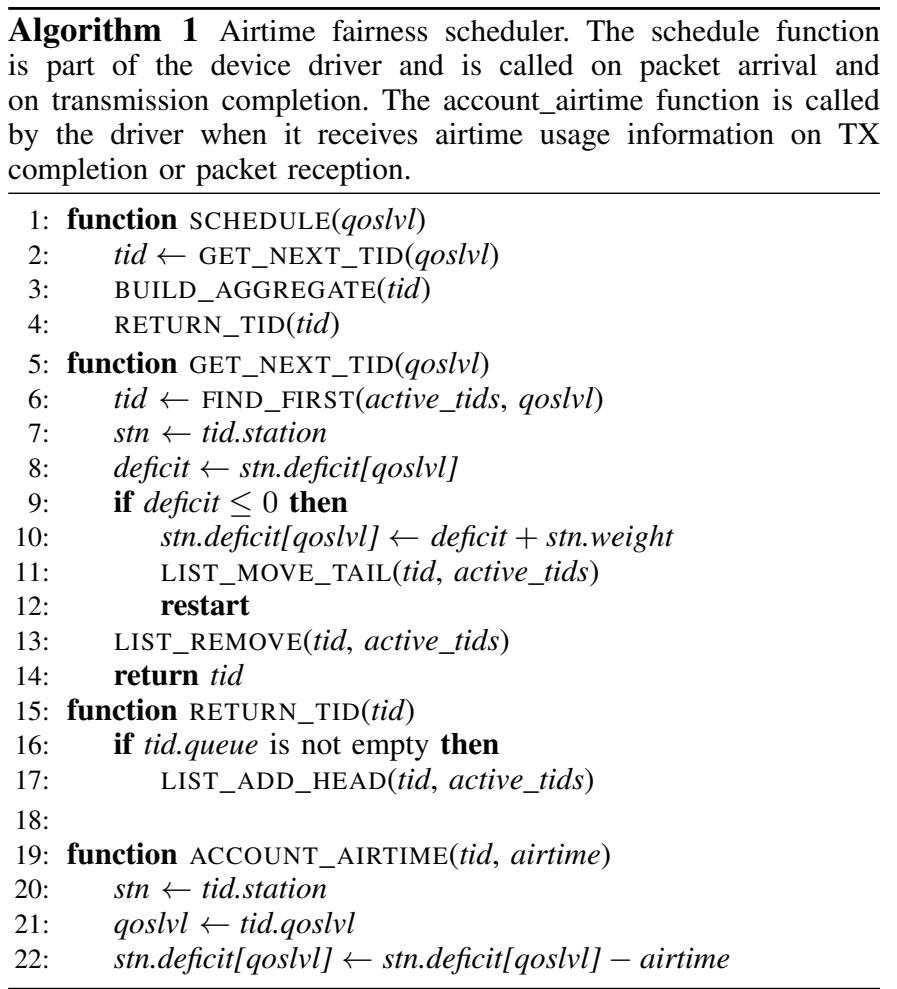

deficit scheduler approach described above. As for the previous airtime fairness scheduler in the driver, airtime deficits are kept separately for each of the four hardware QoS levels, to match the split of the hardware transmission queue scheduling. The algorithm is implemented as part of the get_next_tid() function as shown in Algorithm 1. The PoliFi scheduler has been accepted into the upstream Linux kernel and will appear in Linux 5.1 with support for the ath9k and ath $10 k$ drivers for Qualcomm Atheros 802.11n and 802.11ac hardware.

\section{EVALUATION}

In this section we evaluate how effectively PoliFi is able to implement the desired policies. We examine steady state behaviour as well as the reaction time of the dynamic and limit modes with a changing number of active stations. To show how airtime policies can provide benefits for specific applications, we also include an DASH video streaming use case in our evaluations. We perform the experiments on our testbed with four WiFi devices. The details of our setup are omitted here due to space constraints, but are available in an online appendix [11].

\section{A. Steady state measurements}

The steady state tests consist of running a bulk flow (either UDP or TCP) to each of four stations associated to the access point running PoliFi. Three of the stations are associated to one BSS on the access point, while the fourth is on a separate BSS. These two BSSes are the groups the algorithm balances in dynamic and limit mode. Both groups are given equal weights, meaning that they should receive the same total airtime share. When testing the limit mode use case, the BSS with only 


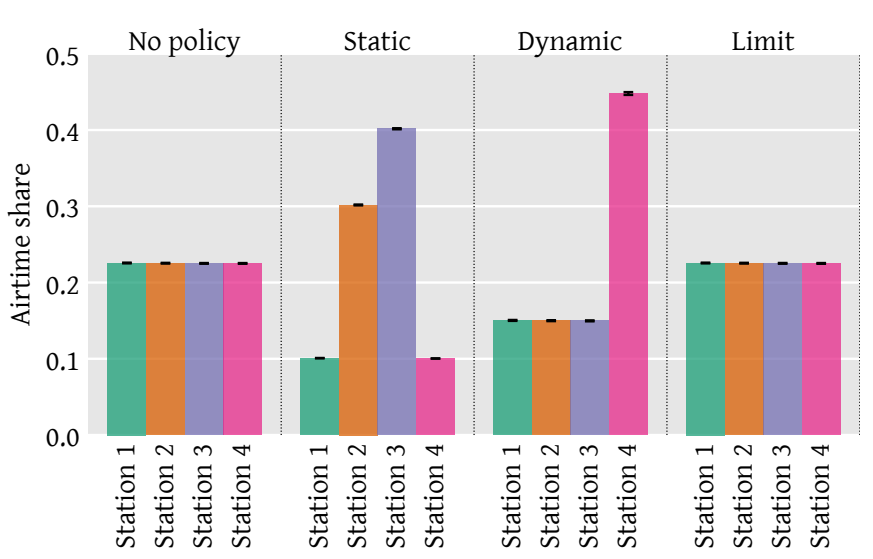

(a) UDP traffic

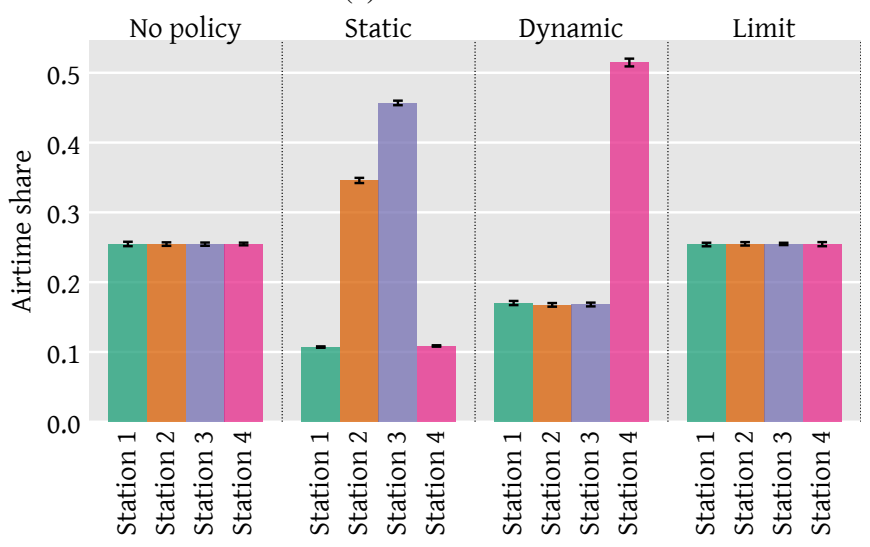

(b) TCP traffic

Fig. 2: Aggregate airtime usage share of four stations, over a 30-second bulk transfer. Graph columns correspond to the different policy modes. In static mode stations 2 and 3 are assigned weights of 3 and 4 , respectively. In dynamic and limit mode, stations 1-3 are on one BSS while station 4 is on another; both BSSes have the same weight, and the second BSS is set to limited. The plots are box plots of 30 test runs, but look like lines due to the low variation between runs.

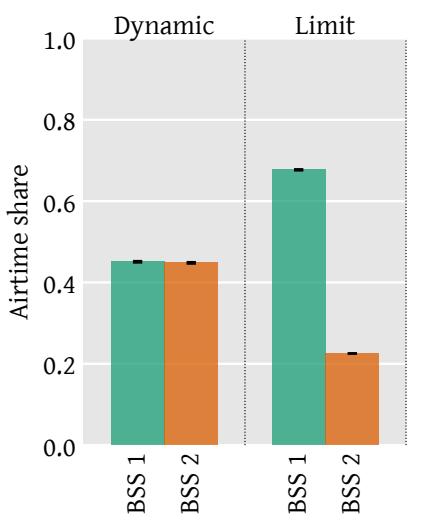

(a) UDP traffic

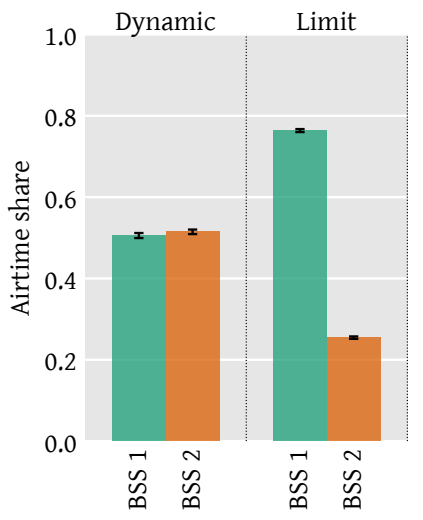

(b) TCP traffic
Fig. 3: Aggregate airtime usage of the two BSSes, for the same test as that shown in Figure 2.

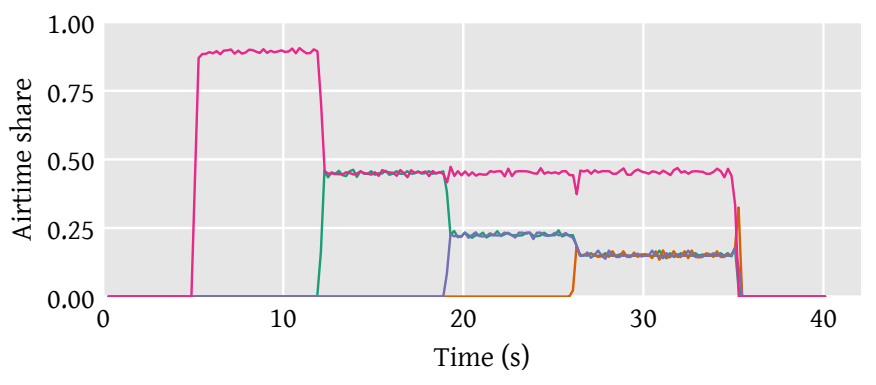

(a) Dynamic mode

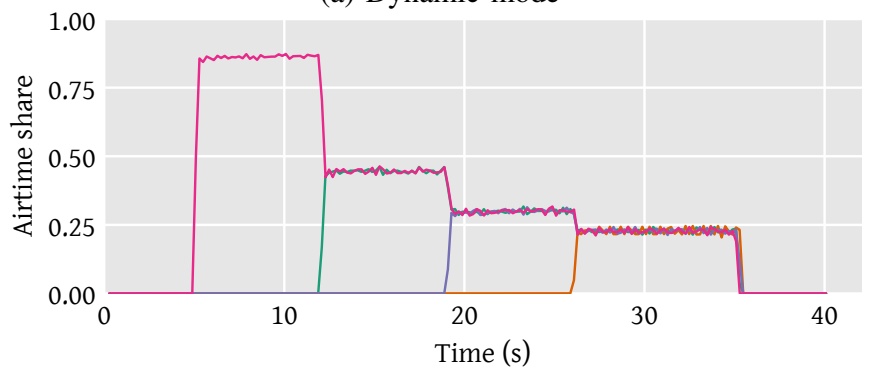

(b) Limit mode

Fig. 4: Airtime usage over time with changing number of active stations, in dynamic and limit mode. UDP flows to each station start 5 seconds apart. The purple station (starting first) is on one BSS, while the remaining three stations are on the other BSS.

a single station in it is set to limited, which in this case means that its natural airtime share is less than the configured share, and thus that no limiting is necessary to enforce the configured policy. We test this to ensure that the algorithm correctly allows the group that is not marked as limited to exceed its configured airtime share.

The aggregate airtime usage of the stations and BSSes is seen in figures 2 and 3 , respectively. With no policy configured, the scheduler simply enforces fairness between the active stations. In the static policy mode, relative weights of 3 and 4 are assigned to stations 2 and 3, respectively. These weights are clearly reflected in the airtime shares achieved by each station in the second column of the graphs in Figure 2, showing that static policy assignment works as designed.

Turning to the group modes, Figure 3 shows the aggregate airtime for each of the two configured BSSes. In dynamic mode, the scheduler enforces equal sharing between the two BSSes, which translates to the single station in BSS 2 getting three times as much airtime as the other three, as is seen in the third column of Figure 2. In limit mode, BSS 2 is limited to at most half of the airtime, but because there is only one station connected to it, its fair share is already less than the limit, and so this corresponds to the case where no policy is enforced. Thus, the tests show that the scheduler successfully enforces the configured policies for all three use cases.

\section{B. Dynamic measurements}

To evaluate the reaction time of the scheduler as station activity varies, we perform another set of UDP tests where 
we start the flows to each of the stations five seconds apart. We perform this test for the dynamic and limit modes, as these are the cases where the scheduler needs to react to changes in station activity.

The results of this dynamic test is shown in Figure 4 as time series graphs of airtime share in each $200 \mathrm{~ms}$ measurement interval. The station that starts first is Station 4 from the previous graphs, i.e., the station that is on BSS 2. In dynamic mode, as seen in Figure 4a, the first station is limited to half the available airtime as soon as the second station starts transmitting. And because the two groups are set to share the airtime evenly, as more stations are added, the first station keeps using half the available airtime, while the others share the remaining half.

In limit mode, as we saw before, the airtime shares of each of the four stations correspond to their fair share. This is also seen in Figure $4 b$, where all stations share the airtime equally as new stations are added.

These dynamic results show that PoliFi has a short reaction time, and can continuously enforce airtime usage policies as station activity changes. This is important for deployment in a real network with varying activity levels.

\section{DASH Traffic Test}

To showcase an example real-world use case that can be improved by airtime policy enforcement, we examine a DASH video streaming application. In this scenario, we add a station with poor signal quality to the network, representing a streaming device that is connected to the wireless network at a location where signal quality is poor. Moving the device is not an option, so other measures are necessary to improve the video quality. We stream the Big Buck Bunny [12] video using the dash.js [13] player running in the Chromium browser on the slow station. We determine that the maximum video bitrate the device can reliably achieve in this scenario (with no competing traffic) is 2 Mbps. However, when the other devices are active, the video bitrate drops to $1 \mathrm{Mbps}$ because of contention.

Figure 5 shows the achieved video bitrate along with the data goodput of the video flow, while three other stations are simultaneously receiving bulk data. With no policy set, the video bitrate drops to $1 \mathrm{Mbps}$, as described above. However, when we prioritise the station (to half the available airtime in this case), the achieved bitrate stays at $2 \mathrm{Mbps}$ throughout the 10-minute video. This shows how PoliFi can improve the performance of a specific real-world application.

\section{CONCLUSION}

We have presented PoliFi, a solution for enforcing airtime usage policies in WiFi networks. Our evaluation shows that PoliFi makes it possible to express a range of useful policies, including prioritisation of specific devices, and balancing or limiting of groups of devices. We have also shown how the policy enforcement can improve the performance of a realworld DASH video streaming application.

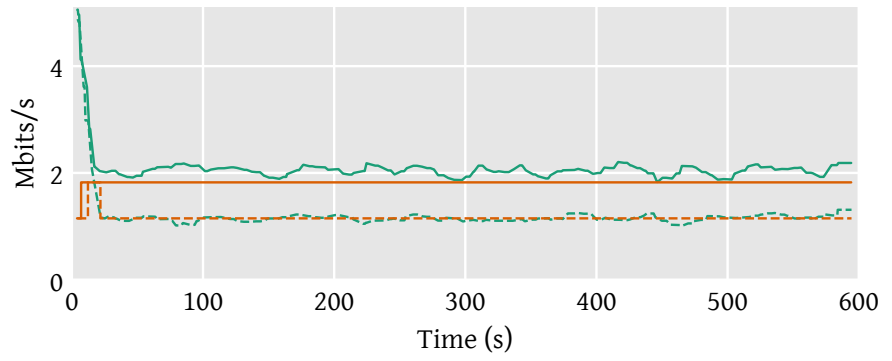

Fig. 5: DASH video throughput with prioritisation (solid lines) and without (dashed lines). The straight lines (orange) show the video bitrate picked by the player, while the others show the actual data stream goodput.

PoliFi can improve performance of WiFi networks with high airtime contention, and enables novel network usages such as network slicing. For this reason we believe it to be an important addition to modern WiFi networks, which is made widely available through its inclusion in the upstream Linux WiFi stack.

\section{REFERENCES}

[1] M. Heusse, F. Rousseau, G. Berger-Sabbatel, and A. Duda, "Performance anomaly of $802.11 \mathrm{~b}$," in IEEE INFOCOM 2003. Twenty-Second Annual Joint Conference of the IEEE Computer and Communications Societies, vol. 2. IEEE, 2003, pp. 836-843.

[2] T. Høiland-Jørgensen, M. Kazior, D. Täht, P. Hurtig, and A. Brunstrom, "Ending the anomaly: Achieving low latency and airtime fairness in wifi," in 2017 USENIX Annual Technical Conference (USENIX ATC 17), 2017.

[3] X. Foukas, G. Patounas, A. Elmokashfi, and M. K. Marina, "Network slicing in 5g: Survey and challenges," IEEE Communications Magazine, vol. 55, no. 5, pp. 94-100, 2017.

[4] J. Babiarz, K. Chan, and F. Baker, "Configuration Guidelines for DiffServ Service Classes," RFC 4594 (Informational), RFC Editor, Aug. 2006, updated by RFC 5865. https://www.rfc-editor.org/rfc/rfc4594.txt

[5] T. Szigeti, J. Henry, and F. Baker, "Mapping Diffserv to IEEE 802.11," RFC 8325 (Proposed Standard), RFC Editor, Feb. 2018. https://www. rfc-editor.org/rfc/rfc8325.txt

[6] M. Richart, J. Baliosian, J. Serrat, J.-L. Gorricho, R. Agüero, and N. Agoulmine, "Resource allocation for network slicing in WiFi access points," in 13th International Conference on Network and Service Management, CNSM, 2017, 2017.

[7] K. Katsalis, K. Choumas, T. Korakis, and L. Tassiulas, "Virtual 802.11 wireless networks with guaranteed throughout sharing," in 2015 IEEE Symposium on Computers and Communication (ISCC), Jul 2015.

[8] Y. Yiakoumis, K.-K. Yap, S. Katti, G. Parulkar, and N. McKeown, "Slicing home networks," in Proceedings of the 2Nd ACM SIGCOMM Workshop on Home Networks, ser. HomeNets '11. ACM, 2011.

[9] "Air Time Fairness (ATF) Phase1 and Phase 2 Deployment Guide," Cisco systems, 2015. https://www.cisco.com/c/en/us/td/docs/wireless/ technology/mesh/8-2/b_Air_Time_Fairness_Phase1_and_Phase2_ Deployment_Guide.html

[10] T. Hoeiland-Joergensen, P. McKenney, D. Taht, J. Gettys, and E. Dumazet, "The Flow Queue CoDel Packet Scheduler and Active Queue Management Algorithm," RFC 8290 (Experimental), RFC Editor, Jan. 2018. https://www.rfc-editor.org/rfc/rfc8290.txt

[11] T. Høiland-Jørgensen, P. Hurtig, and A. Brunstrom, "PoliFi: Airtime Policy Enforcement for WiFi," Feb. 2019. https://doi.org/10.5281/zenodo. 2556784

[12] "Big Buck Bunny," Blender Foundation, 2018. https://peach.blender.org/

[13] "dash.js reference DASH player," Dash Industry Forum, 2018. https: //github.com/Dash-Industry-Forum/dash.js/wiki 